\title{
Estrés, afrontamiento y variables psicológicas intervinientes en el proceso de adaptación a la Lesión Medular (LM): una revisión de la bibliografia
}

\author{
Stress, coping strategies and psychological \\ intervener variables in the adaptacion process to \\ the Medullary Lesion (LM): a bibliographical \\ review
}

\author{
M. ${ }^{\text {a }}$ BEGOÑA RUEDA RUIZ* \\ ANTONIO-LEÓN AGUADO DÍAZ** \\ M. a ÁNGELES ALCEDO RODRÍGUEZ ${ }^{* *}$
}

\section{RESUMEN}

En el siguiente artículo presentamos un recorrido por las investigaciones realizadas en los últimos treinta años sobre el afrontamiento de la LM desde la incorporación de las teorías del estrés en la psicología de la rehabilitación. Hemos revisado la evolución de su concepto en el contexto rehabilitador, los resultados de sus investigaciones así como las dificultades metodológicas existentes para su estudio. El afrontamiento ha demostrado ser una variable de gran relevancia para entender el proceso de adaptación a la LM, en interacción con otras variables psicológicas como las variables de personalidad y el locus de control, ofreciendo apoyo al modelo de las diferencias individuales.

\section{PALABRAS CLAVE}

Lesión medular, Estrés, Estrategias de afrontamiento, Personalidad, Locus de control y adaptación.

* Ayuntamiento de Getxo (Vizcaya).

** Departamento de Psicología, Universidad de Oviedo. 
Estrés, afrontamiento y variables psicológicas intervinientes en el proceso de adaptación a la Lesión Medular (LM)...

\section{ABSTRACT}

This issue presents a review of research about coping with a spinal cord lesion (SCL) in the last thirty years, since the introduction of the stress theory in the rehabilitation psychology. We have reviewed this concept in the rehabilitation context, the research results as well as the methodological limitations for study. Coping has demonstrated to be a very relevant variable to understanding the adaptation process to SCL, in interaction with other psychological variables as personality and locus of control, offering support to the individual differences model.

\section{KEY WORDS}

Spinal cord lesion, Stress, Coping strategies, Personality, Locus of control and adaptation. 


\section{INTRODUCCIÓN: LESIÓN MEDULAR, ESTRÉS Y PROCESO DE ADAPTACIOON}

La explicación del proceso de adaptación a la LM se ha ido desarrollando con las aportaciones de las diferentes teorías psicológicas hasta llegar a la incorporación de los estudios sobre el estrés y el afrontamiento. La evolución del estudio del afrontamiento de la LM es un claro reflejo de la evolución de los modelos teóricos que han existido en psicología de la rehabilitación para entender dicho proceso de adaptación. Caracterizado inicialmente por la presencia de un enfoque psicodinámico y la convergencia teórica con el modelo de las etapas, para ir avanzando hacia la defensa de las diferencias individuales, con la incorporación de las variables cognitivas y el acercamiento a las teorías de personalidad.

Inicialmente el afrontamiento de la LM se identificaba con los mecanismos de defensa y se hablaban de etapas de afrontamiento. Pero a finales de los años ochenta y, principalmente durante los noventa, el estudio del afrontamiento de la LM experimenta un salto cuantitativo y cualitativo, con la incorporación del modelo cognitivo del estrés y la utilización de instrumentos estandarizados para su evaluación. En general, y tomando la utilización de dichos instrumentos como criterio de referencia, diferenciamos un enfoque preempírico y otro empírico en sus investigaciones.

En este sentido, existe una creciente presencia del afrontamiento en los estudios sobre lesionados medulares, desde los trabajos iniciales de Silver y Wortman (1980), Felton y Revenson (1984), a aquellos que introducen el afrontamiento como una variable central de la adaptación a la LM, como Frank, Elliott, Corcoran y Wonderlich (1987), Frank, Umlauf, Wonderlich, Askanazi et al. (1987), Buckelew, Baumstark, Frank y
Hewett, (1990), Buckelew, Frank, Elliott, Chaney et al. (1991), Barone (1993), Hanson, Buckelew, Hewett y O'Neal (1993), Meyer (1998), Soltz (1998), Elfström, Rydén et al (2002), Elfström, Rydén et al (2005), Martz, Livneh, Priebe, Wuermser y Ottomanelli (2005).

Esta mayor presencia va acompañada de una defensa de la bondad del modelo cognitivo del estrés para entender el proceso de adaptación a la LM, en el que el afrontamiento ocupa un lugar central en clara interacción con otras variables psicológicas (Gerhart, Weitzenkamp, Kennedy, Glass y Charlifue, 1999; Galvin y Godfrey, 2001; Pollard y Kennedy, 2007). Dicho modelo quedaría representado en el gráfico de la página siguiente.

Elliott y Frank (1996) sugieren la conveniencia de que las teorias sobre la adaptación a la LM adopten una perspectiva biopsicosocial que tenga en cuenta los efectos interactivos entre la LM, los estresores psicosociales y el binomio personaambiente. Ello permitiría explicar las diferencias individuales, predecir la depresión y otros indicadores de dicha adaptación. Vemos así cómo la incorporación de la perspectiva del estrés y el afrontamiento tiene mucho que aportar al desarrollo del modelo de las diferencias individuales.

Consecuencia directa de la influencia de las teorías del estrés en la psicología de la rehabilitación es la consideración de la LM como un suceso estresante cuya aparición produce muchos cambios en la vida de la persona y le requiere grandes esfuerzos de adaptación. Su naturaleza estresante se deriva de tres elementos principales: las diferentes fuentes de estrés, la naturaleza mixta del suceso estresante considerado como un suceso vital mayor con estresores diarios, $y$, la dimensión objetividad - percepción subjetiva, tal y como se representa en la tabla de la página siguiente. 
Modelo cognitivo del estrés aplicado al proceso de adaptación a la LM (Elaboración propia basada en Galvin y Godfrey, 2001)

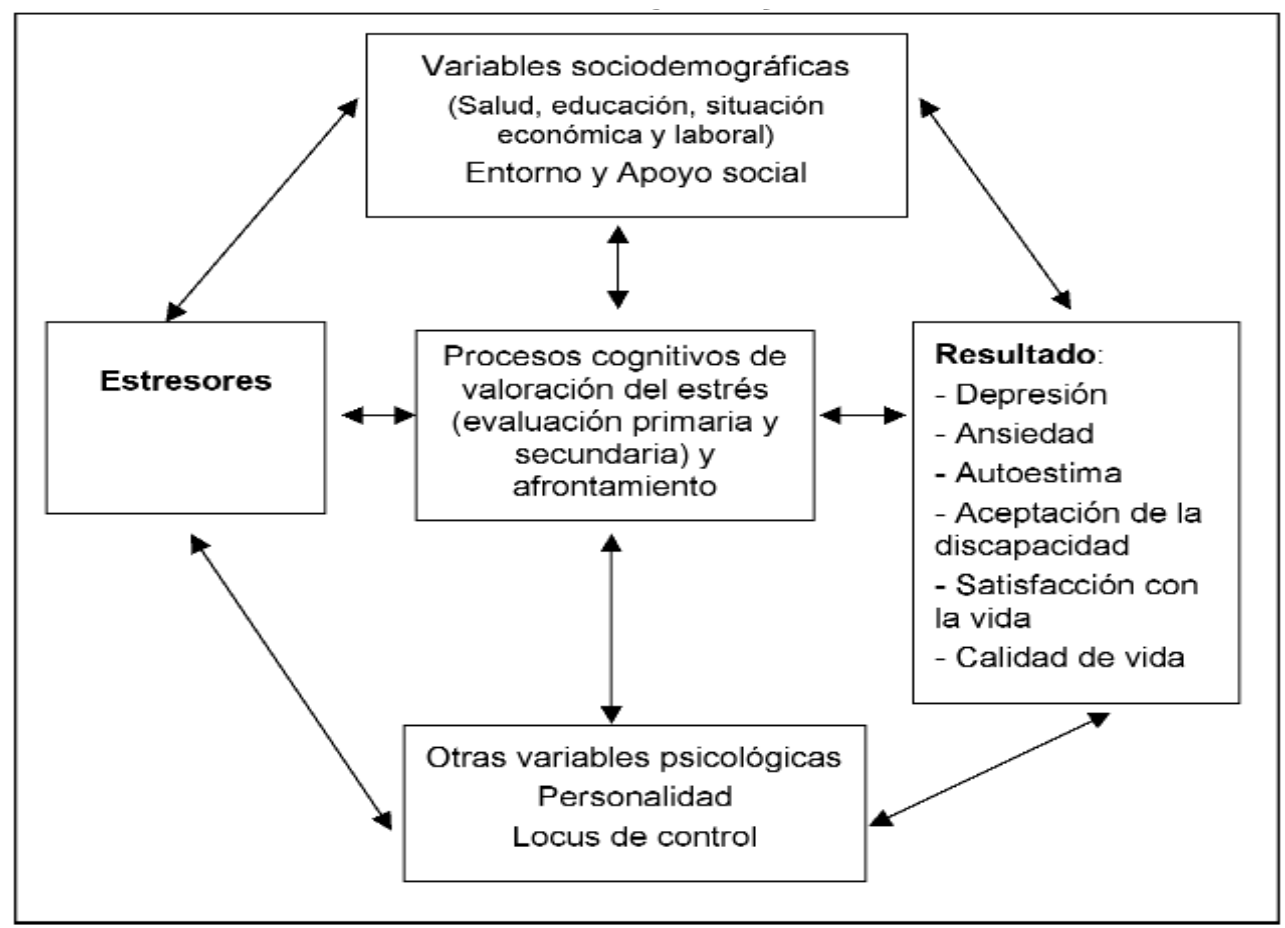

La naturaleza estresante de la LM (Rueda y Aguado, 2003)

\begin{tabular}{|c|c|c|}
\hline & Dimensiones del suceso estresante & Respucsta \\
\hline $\begin{array}{c}\text { Múltiples } \\
\text { Fuentes de estrés }\end{array}$ & $\begin{array}{l}\text { - Cambios orgánicos y fisiológicos. } \\
\text { - } \quad \text { Fntorno hospilalario y tralamientos } \\
\text { médico-rehabilitadores. } \\
\text { - Entorno socio-familiar. }\end{array}$ & $\begin{array}{c}\text { Impacto } \\
\text { Mullidimensional }\end{array}$ \\
\hline $\begin{array}{c}\text { Naluraleza mixla del } \\
\text { succso cstrosantc }\end{array}$ & $\begin{array}{l}\text { - Suceso vilal (alectación severa con grandes } \\
\text { - } \quad \text { Estrós diario (molestias en el descmpeño de } \\
\text { las actividades de la vida diaria). }\end{array}$ & \multirow{2}{*}{$\begin{array}{c}\text { Respucsta } \\
\text { Individual de } \\
\text { ansiedad }\end{array}$} \\
\hline $\begin{array}{c}\text { Dimensión } \\
\text { Objctiva y subjctiva }\end{array}$ & $\begin{array}{l}\text { - Pérdida objeliva de movilidad, sensibilidad } \\
\text { y funcionalidad. } \\
\text { - Valoración subjctiva de la pérdida } \\
\text { (significado personal de la discapacidad). }\end{array}$ & \\
\hline
\end{tabular}


En esta situación nueva que supone la LM, de aparición repentina y frecuentemente traumática, las demandas estresantes pueden provenir de diferentes fuentes como son: su propio organismo y los cambios experimentados ante la aparición de la lesión; la situación hospitalaria y los tratamientos médicos a los que se ve sometida, y el entorno social en el que se encuentra inmersa, siendo de especial relevancia la familia y las actitudes sociales. Todos estos factores deben considerarse desde una perspectiva temporal que se inicia en la fase aguda y continúa a lo largo de la vida.

A pesar de la creciente atención que ha recibido el afrontamiento en el estudio de la LM, existe una falta de coherencia y unanimidad en cuanto a las teorías, su forma de evaluación, los resultados obtenidos, su significado y su papel en el proceso de adaptación. De hecho, los planteamientos existentes en las investigaciones del afrontamiento de la LM adolecen de un modelo teórico apoyado en la evidencia empírica, caracterizándose por un pluralismo teórico y metodológico. En trabajos anteriores hemos revisado dichas cuestiones metodológicas (Rueda y Aguado, 2003). Sin embargo, aunque el estudio del afrontamiento en lesionados medulares no ha sido sistemático y presenta frecuentes limitaciones metodológicas, son muchas las aportaciones realizadas por las teorías del estrés al estudio de los aspectos psicológicos de la LM, tal y como veremos en el siguiente apartado.

\section{APORTACIONES DEL ESTRÉS Y EL AFRONTAMIENTO AL ESTUDIO DEL PROCESO DE ADAPTACIÓN A LA LM}

La aplicación de las teorias del estrés al estudio de la LM y su consideración como una situación estresante tiene implicaciones en el enfoque de estudio del proceso de adaptación, tanto a nivel teórico como clínico y metodológico.

Entre las aportaciones teóricas cabe destacar la despatologización del estudio de la adaptación y de variables clásicas como la depresión, la negación y la personalidad. Así, la adaptación puede explicarse en términos de adecuación entre las demandas de la situación y las capacidades de la persona en interacción con el ambiente, haciendo hincapié en los esfuerzos cognitivos y conductuales, y su eficacia para manejar el estrés. Desde esta perspectiva, la adaptación no se operativiza sólo como la ausencia de depresión, sino que puede incluir mediciones de ansiedad, de emociones positivas, de funcionamiento social, de calidad de vida, de percepción de bienestar y de las estrategias de afrontamiento utilizadas. De igual forma, aparecen conceptos alternativos para explicar la conducta de negación en las personas con LM, como la adaptación cognitiva, la expectativa de recuperación, la negociación con la realidad, la ilusión y la esperanza y las creencias irreales (Rueda y Aguado, 2003). Frente al modelo de las etapas, centrado en buscar la personalidad característica del lesionado medular o los trastornos de personalidad asociados a su aparición, las teorias del estrés dirigen la atención hacia el papel de las variables de personalidad en la utilización de las estrategias de afrontamiento para manejar el estrés. La depresión deja de ser el resultado de la LM y su impacto psicológico, para pasar a considerarse un indicador de la eficacia del afrontamiento.

Este nuevo enfoque tiene implicaciones prácticas y lleva implícito el reconocimiento del protagonismo de la persona en el proceso de adaptación, de tal forma que no es un mero agente pasivo de la sucesión temporal de etapas sino un agente activo en la tarea de optimizar su autonomía personal y su calidad de vida, 
y ello dependerá de su eficacia para manejar el estrés. Consecuentemente, en el área de la intervención clínica, se recupera el papel activo de los profesionales que son agentes rehabilitadores, promotores de estrategias de afrontamiento eficaces y del sentido de control en los pacientes, en su tarea de ofrecer apoyo durante la rehabilitación y facilitar el proceso de adaptación, en consonancia con el trabajo de King y Kennedy (1999).

Junto a las aportaciones teóricas y clínicas que hemos señalado, los estudios del afrontamiento han promovido también la introducción de nuevos instrumentos que permiten avanzar en la operativización del proceso de adaptación a la LM que complementan los cuestionarios tradicionalmente utilizados de depresión y de personalidad.

Para entender estos logros y aportaciones en el estudio de las personas con LM vamos a profundizar en el panorama actual de las investigaciones sobre el afrontamiento de la LM, sus limitaciones metodológicas y los resultados ofrecidos.

\section{ALGUNAS CLAVES PARA ENTENDER EL ESTUDIO DEL AFRONTAMIENTO DE LA LM}

El afrontamiento es un constructo de naturaleza cognitivo-conductual, multidimensional y multicondicionado, que presenta cierta estabilidad por su estrecha relación con las variables de personalidad, a la vez que dinamismo en función de la situación. Podemos hablar de la naturaleza mixta del afrontamiento que incluye cierta estabilidad y un componente situacional en las estrategias utilizadas para manejar el estrés.

En la definición de afrontamiento se incluyen todos los esfuerzos por manejar el estrés, independientemente de los resultados, cuyas funciones principales son la resolución de problemas y el control emocional. Los grandes debates sobre los que ha girado el desarrollo teórico del afrontamiento son: la definición de su constructo y dimensiones, su estabilidad, generalidad vs especificidad, y rasgo vs estado.

Abandonando los planteamientos absolutistas del debate sobre el afrontamiento como rasgo o como proceso, la investigación se debe centrar en analizar hasta qué punto las estrategias de afrontamiento son una función de la persona o del entorno y estudiar la interacción entre ambos. En este sentido, el afrontamiento constituye el resultado de una combinación de disposiciones personales y demandas ambientales, de manera que sólo desde su interacción podemos explicar las diferencias individuales encontradas. Por ello, una comprensión global del afrontamiento requiere el estudio de las estrategias de afrontamiento en relación con las variables de personalidad, así como la realización de estudios específicos de afrontamiento en las diferentes situaciones que aparecen en la vida de una persona con LM.

Gran parte de las investigaciones se ha centrado en el estudio de la eficacia del afrontamiento de la LM y los diferentes intentos de clasificar las múltiples estrategias encontradas. Todos estos aspectos presentan matices que hay que tener en cuenta cuando hablamos del afrontamiento de la LM en el contexto rehabilitador.

En primer lugar, la eficacia del afrontamiento no es una característica intrínseca del propio afrontamiento o de sus estrategias, sino que se construye en la interacción de la persona con el ambiente, presentando gran variabilidad en función de las diferencias individuales, la 
situación y el momento. Es decir, lo que es eficaz para una persona puede no serlo para otra; lo que es eficaz para afrontar una situación concreta no es generalizable a otras situaciones, y lo que resultó eficaz en un momento dado, puede no serlo ahora.

Se suele aceptar que las estrategias de afrontamiento son eficaces si contribuyen al bienestar fisiológico, psicológico y social de la persona. La eficacia de las estrategias de afrontamiento descansa en su habilidad para manejar y reducir el malestar inmediato, así como en sus efectos a largo plazo, en términos de bienestar psicológico y en el estado de salud (Snyder, 1999). La bibliografía al uso muestra cómo los indicadores de la eficacia del afrontamiento de la LM más utilizados han sido el bienestar psicológico y los estados emocionales de ansiedad y depresión.

Dada la naturaleza multicondicionada del afrontamiento, al valorar su eficacia hay que tener en cuenta factores como el área de actividad, en su doble faceta de manejo de la ansiedad emocional y de la situación problema, el momento en el tiempo, a corto y a largo plazo, y el contexto, que incluye las variables del entorno; a lo que hay que añadir la importancia de la percepción subjetiva de dicha eficacia (autoeficacia percibida) y el significado personal de la acción y sus consecuencias.

Todo lo anterior nos remite a la importancia del momento de evaluación en el contexto rehabilitador. Por ejemplo, no es lo mismo el afrontamiento de la LM en la fase aguda de hospitalización que en la fase crónica, en el momento de la rehabilitación hospitalaria que en de la vuelta a casa. Diferentes formas de afrontamiento pueden ser eficaces para manejar los estresores agudos o temporales, pero no los estresores crónicos 0 permanentes, aspectos importantes en nuestra población de estudio. Por todo ello, parece adecuado el estudio especifico del afrontamiento en el entorno rehabilitador y el análisis individualizado de cada persona.

El afrontamiento de la LM se podría estudiar de muchas formas, con un enfoque general o específico. Podemos valorar el afrontamiento de las relaciones interpersonales, de las barreras de accesibilidad o de las dificultades en el trabajo, y pueden utilizarse diferentes estrategias para afrontar sintomas esporádicos 0 intermitentes como el dolor o las infecciones urinarias, y secuelas crónicas como la falta de sensibilidad, la movilidad reducida o la presencia de barreras arquitectónicas. Así, sería interesante investigar el afrontamiento de la LM desde una perspectiva complementaria que contemple tanto el afrontamiento general, a lo largo del tiempo con el afrontamiento especifico, en diferentes situaciones y esferas de la vida. En este sentido, Visser, van den Berg-Emons, Sluis, Bergen, Stam y Bussman (2008) han estudiado las barreras y los elementos facilitadores que las personas con LM se encuentran al finalizar la rehabilitación hospitalaria.

\section{ESTRATEGIAS DE AFRONTAMIENTO UTILIZADAS POR LAS PERSONAS CON LM}

Frente a las afirmaciones iniciales que defendian la existencia de un afrontamiento específico, actualmente se reconoce que la relación univoca entre afrontamiento y discapacidad es muy simplista y que la respuesta a la LM está fuertemente mediatizada por la interacción con otras variables psicológicas. Todo parece apuntar hacia la existencia de diferencias individuales en las estrategias utilizadas por los lesionados medulares para manejar el estrés. 
Existe evidencia de que las estrategias de afrontamiento utilizadas por los lesionados medulares no dependen del nivel ni del tipo de lesión, sino de patrones de afrontamiento diversos que se pueden explicar en función de otras variables, pero no por la lesión en sí misma (Frank, Umlauf et al., 1987; Buckelew et al., 1990; Reidy et al., 1991; Hancock et al., 1993; Kennedy et al., 1995 ; Elfström, Kreuter et al., 2002 ; Rueda y Aguado, 2003; Elfström et al., 2005). La etiologia, por su parte produce algunas diferencias leves en el afrontamiento actual, y el impacto en el momento de aparición parece ser mayor en las LM altas independientemente de que sean completas 0 incompletas. En este sentido, la naturaleza traumática de la LM parece influir en la "aceptación -conformismo y serenidad", siendo las de origen deportivo las que mayores puntuaciones presentan en esta estrategia. La presencia de complicaciones médicas parece asociarse a una peor adaptación en el momento actual, reflejada en una menor "aceptación" y una mayor "inevitabilidad con futuro negativo” (Rueda y Aguado, 2003).

El análisis del papel de las variables sociodemográficas parece sugerir que no hay diferencias de afrontamiento en función del sexo, los jóvenes parecen adaptarse mejor, el impacto de la LM y el papel de la familia parecen ser mayores en función de la edad. La influencia del estado civil parece ser mayor en el momento de aparición de la LM y la presencia de pareja para explicar algunas diferencias. Respecto al nivel de estudios es la ausencia de estudios el factor que produce diferencias que se reflejan en el uso de las estrategias de manejo de la información.

En general, los estudios del afrontamiento de la LM se han centrado en la búsqueda de las estrategias utilizadas por este colectivo y, principalmente, en el análisis de la eficacia de dichas estrate- gias en el proceso de adaptación, descuidando el estudio de los instrumentos de evaluación del afrontamiento y su adecuación para esta población. Los estudios sobre el afrontamiento de la LM confirman la tendencia a la diversidad de las estrategias utilizadas y la falta de consenso sobre su descripción y clasificación.

Entre las conductas de afrontamiento más eficaces y asociadas con un mayor bienestar en las personas con LM se encuentran la resolución de problemas, la planificación y la reestructuración cognitiva (Buckelew et al., 1990; Elliott, Godshall et al., 1991; Hanson et al., 1993) y la búsqueda de apoyo social (Coca, 1990; Reidy et al., 1991; Moore et al., 1994; Kennedy et al., 1995). Junto a éstas, la revaloración positiva, el distanciamiento, el autocontrol y la confrontación (Warner, 1989; Reidy et al., 1991; Barone, 1993; Dias de Carvalho et al., 1998; Carroll, 1999), así como la aceptación (Kennedy et al., 1995; Dias de Carvalho et al., 1998; Elfström, Kreuter et al., 2002), la aceptación de la responsabilidad (Reidy et al., 1991) y el espíritu de lucha (Elfström, Kreuter et al., 2002) parecen de utilidad para afrontar la LM. La búsqueda de información también es eficaz, aunque según el momento en el proceso de adaptación, siendo más adaptativa transcurridos unos meses después de la lesión (Buckelew et al., 1990), en consonancia con los resultados de Felton et al. (1984) sobre el afrontamiento de las enfermedades crónicas.

Por otro lado, las estrategias de afrontamiento menos eficaces y asociadas a una mayor depresión o ansiedad son las conductas de escape-evitación (Warner, 1989; Coca, 1990; Reidy et al., 1991; Barone, 1993; Moore et al., 1994; Meyer, 1998; Carroll, 1999), la minimización de la amenaza (Buckelew et al., 1990, 1991), la desvinculación conductual (Ken- 
nedy et al., 1995), el distanciamiento mental y la supresión de actividades (Lude, Kennedy, Evans, Lude y Beedie, 2005), el pensamiento desiderativo (Frank et al., 1987; Buckelew et al., 1990, 1991; Hanson et al., 1993; Moore et al., 1994), la dependencia social (Elfström, Kreuter et al., 2002), la confrontación y la culpabilización (Dias de Carvalho et al., 1998), así como la preocupación mental por la causa de la lesión y su evitabilidad (Van Den Bout et al., 1988)

Además, se ha observado que un alto nivel de esperanza y cierto grado de optimismo pueden ser eficaces en la adaptación a la LM si se combinan con estrategias de afrontamiento centradas en el problema y en la búsqueda de soluciones, de ayuda, consejo o apoyo social (Moore et al., 1994).

Englobando los resultados anteriores, existe bastante consenso entre los autores al afirmar que, en general, el afrontamiento activo y centrado en la resolución de problemas está asociado a menores indices de depresión, mientras que el afrontamiento pasivo, que incluye principalmente las estrategias de evitación o centradas en la emoción, suele ir acompañado de mayor malestar psicológico (Frank et al., 1987; Coca, 1990; Buckelew et al., 1990, 1991; Elliott, Godshall et al., 1991; Reidy et al., 1991; Barone, 1993; Moore et al., 1994; Kennedy et al., 1995; Meyer, 1998; Soltz, 1998; Carroll, 1999; Rueda y Aguado, 2003, Elfström et al, 2005). Sin embargo, también influye la posibilidad de modificar la situación. Así, cuando la persona considera que la situación es susceptible de cambio tiende a utilizar estrategias de afrontamiento activo, como la planificación, la supresión de actividades competitivas o la búsqueda de apoyo social instrumental; mientras que si considera que no puede intervenir, aumenta la utilización de las estrategias centradas en la emo- ción, la aceptación y la negación. A la luz de estos resultados cobran protagonismo variables como el locus, la valoración de los recursos y la autoeficacia percibida.

Las implicaciones derivadas de estos resultados para la psicologia de la rehabilitación sugieren la conveniencia de promocionar la utilización de determinadas estrategias de afrontamiento que son eficaces. King y Kennedy (1999) ofrecen datos preliminares sobre la utilidad de programas de entrenamiento del afrontamiento para el proceso de adaptación en personas con LM.

Sin embargo, hay que distinguir la eficacia del afrontamiento a corto plazo de su eficacia a largo plazo. Los estudios generales del afrontamiento sugieren que la importancia de algunas estrategias de afrontamiento cambia con el tiempo, apoyando la idea del afrontamiento como un proceso dinámico que se va modificando para responder a las necesidades cambiantes de la persona. En este sentido, hay evidencia de que con el paso del tiempo las estrategias centradas en la emoción, la búsqueda de información y de reestructuración cognitiva se asocian con un mayor bienestar en personas con LM (Buckelew et al., 1990; Hanson et al., 1993). Los lesionados medulares agudos tienen más probabilidad de utilizar las estrategias de apoyo social (tanto emocional como instrumental). En nuestra investigación hemos encontrado un afrontamiento actual más caracterizado por estrategias activas y de resolución de problemas, mayor aceptación y reestructuración cognitiva; y un afrontamiento rememorado en el que predominan las estrategias de corte emotivo y existe una mayor negación, a la vez que coexisten las estrategias de resolución de problemas y las cognitivas (Rueda y Aguado, 2003).

En este sentido, a pesar de la evidencia de una dinámica del afrontamiento 
con el paso del tiempo, la comparación del afrontamiento actual y rememorado ha revelado un alto porcentaje de estrategias que son comunes a lo largo del proceso de adaptación a la LM. Todo parece apuntar a que las estrategias centradas en la emoción son reflejo del grado de adaptación y de los niveles de estrés, mientras que las estrategias de resolución de problemas, la búsqueda de información, la reestructuración cognitiva, las conductas de interacción social, el olvido como alejamiento, los pensamientos reconfortantes, la religión o un ideal de vida son útiles para manejar el estrés, independientemente del tiempo transcurrido desde la lesión. Por lo tanto hablamos de una dinámica en las estrategias de afrontamiento utilizadas en función del curso de la rehabilitación y del paso del tiempo, junto a cierta estabilidad, defendida también por autores como Hanson et al. (1993) y Pollard y Kennedy (2007).

La variable edad en el momento de aparición de la LM es otro factor a tener en cuenta para entender la eficacia del afrontamiento. Diversos autores han sugerido que las personas jóvenes con LM suelen utilizar estrategias de afrontamiento diferentes a las de los lesionados medulares de edad avanzada (Galvin y Godfrey, 2001; Elfström, Kreuter et al., 2002). No parece descabellado pensar que en el transcurso vital, el lesionado medular puede realizar cambios en su afrontamiento en función de las diferentes situaciones a las que se enfrenta y sus necesidades en ese momento.

Junto a la multidimensionalidad hay que destacar la bidireccionalidad de la relación afrontamiento-depresión y su naturaleza interactiva, tal como se ha sugerido (Buckelew et al., 1990; Kennedy et al. 1995 y Galvin y Godfrey, 2001). Las estrategias de afrontamiento utilizadas, los resultados que producen y la valoración de esos resultados influyen en la utilización posterior de esas u otras estrategias, en consonancia con el modelo cognitivo del estrés que mencionábamos en apartados anteriores.

Los análisis de las puntuaciones de depresión en nuestra investigación confirman esa relación bidireccional entre el afrontamiento y la depresión de tal forma que el grado de depresión no se interprete únicamente como indicador de la eficacia del afrontamiento, sino como un factor que también puede influir en las estrategias utilizadas. Así, hemos encontrado estrategias que se relacionan con el grado de depresión y que son reflejo del estado emocional. Por un lado, tenemos la "inevitabilidad con futuro negativo", el "impacto intenso y negativo de enfermedad" en sentido negativo y, por otro, la "aceptación, conformismo y serenidad", el "enganche con la vida con hedonismo" o la "entereza con siempre es posible un peor" en sentido positivo.

Como venimos defendiendo a lo largo de este artículo, las diferencias de afrontamiento encontradas en las personas con LM hay que entenderlas en interacción con otras variables psicológicas como el locus de control y las variables de personalidad. Así, hay evidencia que apoya la existencia de diferencias individuales en las estrategias de afrontamiento utilizadas en función de las expectativas de control de los lesionados medulares (Frank, y Elliott, 1987; Frank, Umlauf et al., 1987; Buckelew et al., 1990; Barone, 1993; Hancock et al., 1993; Craig et al., 1994; Rueda y Aguado, 2003).

Las personas con LM con un locus interno se adaptan mejor, tienen mayor eficacia percibida y utilizan estrategias de afrontamiento activo; mientras que las personas con locus externo utilizan más las estrategias de pensamiento de- 
siderativo, el afrontamiento mixto $y$ de crecimiento y la autoculpa. De hecho, la confianza de los lesionados medulares en sus estrategias de resolución de problemas se relaciona con la realización de autocuidados y una menor incidencia de complicaciones secundarias a la LM (Richards et al., 2002).

También se ha demostrado la relación entre el afrontamiento y las variables de personalidad E-N, en estrecha relación con el locus. Así, E y el locus interno se relacionan con estrategias activas y de resolución de problemas, mientras que $\mathrm{N}$ y el locus externo se asocian con estrategias centradas en la emoción, que reflejan un mayor malestar psicológico y un mayor impacto de la LM (Rueda y Aguado, 2003).

La importancia del afrontamiento en la rehabilitación y adaptación a la LM queda reflejada en la diversidad de temas con los que se ha investigado. Algunos estudios han abordado el estudio del afrontamiento de la LM y su relación con el dolor (Raichle, Hanley y Jensen, 2007), el estrés postraumático (Lude et al., 2005), la resistencia personal (Kinder, 2005), adaptación de pareja y carga de la persona cuidadora (Chan, Lee y Lie-Mak, 2000), percepción del paciente y los profesionales (Siösteen, Kreuter, Lampic y Persson, 2005).

\section{DIFICULTADES PARA EL ESTUDIO DEL AFRONTAMIENTO DE LAS PERSONAS CON LM}

El análisis de las investigaciones revisadas revela la existencia de frecuentes deficiencias metodológicas en los estudios del afrontamiento de la LM, entre las que cabe destacar la composición de la muestra, la presencia de variables de confundio, el momento de evaluación, junto a la frecuente presencia de diseños deficitarios en las investigaciones con ausencia de una metodologia diferencial y de estudios longitudinales, y una utilización de instrumentos de escasa validez para la LM.

Así, ante las dificultades de captación de la muestra, es frecuente la realización de los estudios con muestras de conveniencia pequeñas y heterogéneas, obtenidas frecuentemente durante la fase de hospitalización, que no consideran aspectos relevantes como son la edad, la etiología de la LM y otras variables sociodemográficas. La única variable que suele estar bien representada es el sexo, con una mayor presencia de hombres (80\% de hombres y $20 \%$ de mujeres). Respecto a la etiología de la LM, existe un predominio de investigaciones de la LM traumática, sin apenas presencia de LM de etiologia tumoral o secundaria a otros procesos patológicos. La mayoría de los estudios no controlan la existencia de psicopatología previa a la lesión, la presencia de dificultades económicas, las variables médicas y terapéuticas (reingresos y complicaciones médicas), del entorno de procedencia y el apoyo familiar.

Respecto al tamaño de las muestras, es frecuente la realización de los estudios con muestras pequeñas, que pueden variar desde los 6 sujetos de Pereira y Araujo (2005) hasta los 257 de Wineman, Duran y Steiner (1994), siendo la composición media de la muestra de las investigaciones alrededor de los 50 sujetos. Excepto los estudios que consiguen valorar a más de cien lesionados medulares, las demás investigaciones se realizan con muestras pequeñas y a menudo poco representativas.

El tiempo de evolución desde la aparición de la lesión presenta también gran variabilidad, predominando la investigación durante la hospitalización y en los 
primeros dieciocho meses desde la aparición de la lesión. En general, los estudios de evaluación única tienen una mayor presencia de muestras de lesionados medulares de larga evolución (desde dos años hasta veintidos años después de la LM), mientras que las investigaciones que realizan varias evaluaciones han elegido preferentemente muestras de lesionados medulares agudos, ya sea durante la hospitalización 0 en periodos cercanos al alta. Algunos autores incluyen de forma indiferenciada lesionados medulares agudos y no agudos en las muestras. A este respecto, hay que considerar las dificultades derivadas de comparar grupos de LM agudos y de larga evolución, asumiendo que representan muestras de una misma población en diferentes momentos (por las variables debidas al curso de la rehabilitación y las posibles complicaciones médicas, así como posibles estresores concurrentes en la fase aguda, como la pérdida de seres queridos en el accidente, la incertidumbre sobre el futuro laboral, la situación económica, la reacción de la familia, etc).

Existe una escasa frecuencia de estudios de seguimiento del afrontamiento de la LM y la mayoría de los estudios son correlacionales, realizados durante la fase aguda de la rehabilitación. Así mismo, la relación entre el afrontamiento y la adaptación se ha estudiado principalmente a través de estudios transversales de evaluación única y son pocos los estudios que tienen un diseño de multievaluación de seguimiento. Como hemos visto anteriormente el afrontamiento y las variables relacionadas con su eficacia en la fase aguda pueden ser bastante diferentes que en otros momentos de la rehabilitación y que la utilidad de las estrategias cambia con el tiempo. En cualquier caso, son necesarias futuras investigaciones que combinen el estudio transversal de lesionados medulares agudos y de larga evolución con estudios longitudinales de seguimiento que realicen mediciones objetivas del afrontamiento a lo largo del tiempo.

En este sentido, destacables son los los estudios de McColl y Skinner (1995) que realizaron tres mediciones en doce meses, de Craig et al. (1994) que evaluaron tres veces en un periodo de dos años, y de Hancock et al. (1993), quienes realizaron cuatro evaluaciones. Un trabajo reciente de Pollard y Kennedy (2007) ha hecho seguimiento al cabo de 10 años después del alta.

En ocasiones anteriores hemos revisado los instrumentos utilizados para la evaluación del afrontamiento en personas con LM (Rueda y Aguado, 2003; Rueda, Aguado y Alcedo, 2004). La tendencia general ha sido la utilización de cuestionarios generales de afrontamiento, la aplicación de algunos cuestionarios construidos en el área de la salud y la ausencia de instrumentos específicos para evaluar el afrontamiento de la LM. Existe un predominio absoluto del instrumento creado por Folkman y Lazarus, el WOC-Ways of Doping. Otros instrumentos generales de afrontamiento utilizados en personas con LM han sido el COPE y el CSQ-Coping Strategies Questionnaire. También se han utilizado instrumentos del área de la salud para evaluar el afrontamiento de la LM, cuya validez para la población con LM es cuestionable, el MAC-Mental Adjustment to Cancer y el Coping Assessment Scale, construidos para poblaciones clinicas.

Sin embargo, las divergencias en la operativización del afrontamiento de la LM plantea la necesidad de llegar a una definición única del afrontamiento y adecuar los instrumentos de medición de la misma, de tal forma que tengan validez para su estudio en el entorno rehabilitador y sean sensibles a las características 
de la propia LM. Es necesario saber qué entendemos por afrontamiento de la LM y cómo vamos a medirlo, para lo cual, un requisito previo es la realización de estudios de validación de los instrumentos generales de afrontamiento para la población con LM y la construcción de instrumentos específicos.

En este sentido, contamos con experiencias de construcción de escalas específicas para la evaluación de personas con LM, como son los trabajos del equipo de Aguado cuya Escala Multidimensional de Evaluación de Lesionados medulares EMELM (Aguado y Alcedo, 1999) incluye dos cuestionarios de afrontamento actual y rememorado (Rueda, 2001; Aguado y Rueda, 2003); o el trabajo del equipo de Elfström de creación de escalas específicas para la evaluación de afrontamiento, consecuencias psicológicas (Elfström, Rydén et al. (2002), o calidad de vida en personas con LM (Elfström et al., 2005).

\section{CONCLUSIONES}

En general, podemos afirmar la creciente presencia del afrontamiento en los estudios de la LM, a pesar de que la psicologia de la rehabilitación no ha realizado una aplicación sistemática de las teorías del estrés al estudio de la LM, ni existe una modelo elaborado que integre la variable afrontamiento y explique su papel dentro del proceso de adaptación. Se reconoce su importancia dentro de las variables psicológicas pero sin una clara delimitación conceptual ni una operativización consensuada. La bibliografía muestra las frecuentes deficiencias metodológicas en su estudio, a la vez que ofrece evidencia que apoya la importan- cia de esta variable en interacción con otras variables psicológicas.

Los datos anteriormente expuestos apoyan las siguientes conclusiones sobre el afrontamiento de las personas con LM:

- No hay un afrontamiento específico utilizado por las personas con LM, sino que hablamos de diferencias individuales.

- El afrontamiento no se relaciona con las variables clínicas. Sin embargo, las variables psicológicas, y entre ellas el afrontamiento, tienen gran poder predictivo de la adaptación a la LM.

- Hay diferencias entre el afrontamiento actual y el rememorado, por lo que parece existir cierta dinámica con el paso del tiempo.

- Junto a esta naturaleza dinámica, parece existir ciertas tendencias estables en las estrategias de afrontamiento utilizadas, relacionadas con las variables de personalidad

- Aunque hay diferencias individuales en el afrontamiento utilizado, algunas estrategias han mostrado ser más eficaces que otras.

Para concluir, podemos decir que el afrontamiento de la LM constituye una linea esperanzadora de investigación que debe seguir avanzando tanto en la elaboración teórica de un modelo coherente que lo ubique dentro de la psicologia de la rehabilitación y sus variables tradicionales de estudio, como en la elaboración o adaptación de instrumentos válidos para su medición. 


\section{BIBLIOGRAFÍA}

Aguado, A.L. y Alcedo, M.A. (1999): "Evaluación psicológica en rehabilitación”. En Ruano, A., Muñoz, J.M. y Cid, C. (Coords.): Psicología de la rehabilitación en deficiencias y discapacidades fisicas, (pp. 33-58). Madrid: Fundación Mapfre Medicina.

Barone, S.H. (1993): Adaptation to spinal cord injury (Coping, Roy Adaptation Model). Tesis doctoral, Boston College (Dissertation Abstracts International, vol. 54-7B, pág. 3547).

Buckelew, S.P., Baumstark, K.E., Frank, R.G. y Hewett, J.E. (1990): "Adjustment following Spinal Cord Injury”. Rehabilitation Psychology, 35 (2), 101-109.

Buckelew, S.P., Frank, R.G., Elliott, T.R., Chaney, J. y Hewett, J. (1991): "Adjustment to spinal cord injury: Stage theory revisited”. Paraplegia, 29 (2), 125-130.

Carroll, A. (1999): The relationship between control beliefs, coping strategies, time since injury and quality of life among persons with spinal cord injuries. Tesis doctoral, Universidad de Kent (Dissertation Abstracts International, vol. 60-6B, pág. 2934).

Coca, B. (1990): Coping strategies, social sup port and acceptance of disability among persons with spinal cord injury. Tesis doctoral, Universidad de Los Angeles. (Dissertation Abstract, vol. 5111B, pág. 5569).

Chan, R.C.; Lee, P.W. y Lieh-Mak, F. (2000): "Coping with spinal cord injury: personal and marital adjustment in the Hong Kong Chinese setting". Spinal Cord, 38 (11), 687-96.

Craig, A.R., Hancock, K.M. y Chang. E. (1994): "The influence of spinal cord injury on coping styles and self-perceptions two years after the injury". Australian and New Zealand Journal of Psychiatry, 28 (2), 307-312.

Dias de Carvalho, S.A., Andrade, M.J., Tavares, M.A. y Sarmento de Freitas, J.L. (1998): "Spinal cord injury and psychological response". General Hospital Psychiatry, 20, 353-359.

Elfström, M.L.; Kreuter, M.; Rydén, A.; Persson, L.O. y Sullivan, M. (2002): "Effects of coping on psychological outcome when controlling for background variables: a study of traumatically spinal cord lesioned persons". Spinal Cord, 40, 408-415.

Elfström, M.L.; Rydén, A.; Kreuter, M.; Persson, L.O. y Sullivan, M. (2002): "Linkages between coping and psychological outcom in the spinal cord lesioned: development of SCL-related measures". Spinal Cord, 40, 23-29.

Elfström, M.L.; Rydén, A.; Kreuter, M.; Taft, C. y Sullivan, M. (2005): "Relations between coping strategies and health-related quality of life in patients with spinal cord lesion". Journal of Rehabilitation Medicine, 37, 9-16.

Elfström, M.L.; Kennedy, P.; Lude, P. y Taylor, N. (2007): "Condition-related coping strategies in persons with spinal cord lesion: a cross-national validation of the Spinal Cord Lesion-related Coping Strategies Questionnaire in four community samples”. Spinal Cord, 45, 420-428.

Elliott, T.R., y Frank, R.G. (1996): "Depression following spinal cord injury". Archives of Physical Medicine and Rehabilitation, 77 (9), 816-823.

Elliott, T.R., Godshall, F.J., Herrick, S.M., Witty, T.E. y Spruell, M. (1991): "Problem-solving appraisal and psychological adjustment following spinal cord injury". Cognitive Therapy and Research, 15 (5), 387-398.

Felton, B.J. y Revenson, T.A. (1984): "Coping with chronic illness: A study of illness controlability and the influence of coping strategies on psychological adjustment". Journal of Consulting and Clinical Psychology, 52 (4), 343-353.

Frank, R.G., Elliott, T.R., Corcoran, J.R. y Wonderlich, S.A. (1987): "Depression after spinal cord injury: Is it necessary?". Clinical Psychology Review, 7 (1), 1-20.

Frank, R.G., Umlauf, R.L., Wonderlich, S.A., Ashkanazi, G.S., Buckelew, S.P. y Elliott, T.R (1987): "Differences in coping styles among persons with spinal cord injury: A cluster-analytic approach". Journal of Consulting and Clinical Psychology, 55 (5), 727-731.

Galvin, L.R. y Godfrey, H.P.D. (2001): "The impact of coping on emotional adjustment to spinal cord injury (SCI): review of the literature and application of a stress appraisal and coping formulation”. Spinal Cord, 39, 615-627. 
Gerhart, K.A: Weitzenkamp, D.A.; Kennedy, P. Glass, C.A. y Charlifue, S.W. (1999): "Correlates of stress in long.term spinal cord injury". Spinal Cord, 37, 183-190.

Hancock, K.M., Craig, A.R., Tennant, C. y Chang. E. (1993): "The influence of spinal cord injury on coping styles and self-perceptions: A controlled study". Australian and New Zealand Journal of Psychiatry, 27 (5), 450-456.

Hanson, S., Buckelew, S.P., Hewett, J. y O Neal, G. (1993): "The relationship between coping and adjustment after spinal cord injury: A 5year follow-up study". Rehabilitation Psychology, 38 (1), 41-52

Kennedy, P.; Lowe, R.; Grey, N., y Short, E. (1995): "Traumatic spinal cord injury and psychological impact: A cross-sectional analysis of coping strategies". British Journal of Clinical Psychology. 34 (4), 627-639.

Kennedy, P. et al. (2000): "A longitudinal analysis of psychological impact and coping strategies following spinal cord injury". British Journal of Health Psychology, 5, 157-172.

Kinder RA. (2005): "Psychological hardiness in women with paraplegia”. Rehabilitation Nursing, 30 (2), 68-72

King, C.A. y Kennedy, P. (1999): "Coping effectiveness training for people with spinal cord injury: Preliminary results of a controlled trial". British Journal of Clinical Psychology, 38 (1), 5-14.

Lude, P.; Kennedy, P.; Evans, M.; Lude, Y. y Beedie, A. (2005): "Post traumatic distress symptoms following spinal cord injury: a comparative review of European samples". Spinal Cord, 43 (2), $102-8$

Martz, E.; Livneh, H.; Priebe, M.; Wuermser, L.A. y Ottomanelli, L. (2005): "Predictors of psychosocial adaptation among people with spinal cord injury or disorder". Archives of Physical Medicine and Rehabilitation, 86 (6),1182-92.

McColl, M.A. y Skinner, H.A. (1995): "Assessing inter and intrapersonal resources: Social support and coping among adults with a disability". Disability and Rehabilitation, 17 (1), 24-34.

Meyer, T.M. (1998): Coping and adjustment during acute rehabilitation for spinal cord injury. Tesis doctoral, Universidad de Auburn. (Dissertation Abstracts International, vol. 59-08B, pág. 4474).
Moore, A.D., Bombardier, C.H., Brown, P.B. y Patterson, D.R. (1994): "Coping and emotional attributions following spinal cord injury”. International Journal of Rehabilitation Research, 17 (1), 3948.

Pereira, M.E. y Araujo, T.Z. (2005): "Estratégias de enfrentamiento na reabilitaçao do traumatismo raquimedular". Arq Neuropsiquiatr, 63 (2-B), 502507.

Pollard, C. y Kennedy, P. (2007): "A longitudinal analysis of emotional impact, coping strategies and post-traumatic psychological growth following spinal cord injury: a 10-year review". British Journal of Health Psychology, 12 (3), 347-62.

Raichle, K.A.; Hanley, M.; Jensen, M.P. y Cardenas, D.D. (2007): "Cognitions, coping, and socia environment predict adjustment to pain in spinal cord injury". Journal of Pain, 8 (9), 718-29.

Reidy, K., Caplan, B. y Shawaryn, M. (1991): "Coping strategies following spinal cord injury: Accomodation to trauma and disability". Comunicación presentada en el 68 Encuentro Anual del Congreso Americano de Rehabilitación, Washington, D.C.

Richards, J.S.; Kewman, D.G., y Pierce, C.A. (2002): Spinal cord injury. En R. G. Frank y T. R. Elliott (Eds.): Handbook of Rehabilitation Psychology, $2^{\text {a }}$ edición (pp. 11-28). Washington: American Psychological Association.

Rueda, M.B. (2001): Evaluación del afrontamiento en Lesionados Medulares. Tesis doctoral, Universidad de Deusto (Edición electrónica № 3029788). Madrid: Bell \& Howell España (ISBN: 84-7485-776-7)

Rueda, M.B. y Aguado, A.L. (2003): Estrategias de afrontamiento y proceso de adaptación a la lesión medular. Madrid, IMSERSO.

Rueda, M.B.; Aguado, A.L. y Alcedo, M.A. (2004): "Evaluación del afrontamiento de las personas con lesión medular". Análisis y modificación de conducta, 30 (130), 273-310.

Silver, R.L. y Wortman, C.B. (1980): "Coping with undesirable life events". En J. Garbey y M. Seligman (Eds.): Human helplessness: Theory and applications, (pp. 279-375). Nueva York: Academic Press.

Siösteen, A.; Kreuter, M.; Lampic, C. y O Persson, L, (2005): "Patient-staff aoreement in the perception of spinal cord lesioned patients' problems, 
Estrés, afrontamiento y variables psicológicas intervinientes en el proceso de adaptación a la Lesión Medular (LM)..

emotional well-being, and coping pattern”. Spinal Cord, 43, 179-186.

Soltz, D.R. (1998): The effects of acquired disability on coping with the disability. Tesina de Master, Universidad de California (Master Abstracts International, vol. 36-06B, pág. 1445).

Snyder, C.R. (Ed.) (1999): Coping. The psychology of what works. Nueva York: Oxford University Press.

Van Den Bout, J., Van Son-Schoones, N, Schipper, J. y Groffen, C. (1988): "Attributional cognitions, coping behavior and self-esteem in inpatients with severe spinal cord injuries". Journal of Clinical psychology, 44 (1), 17-22.
Vissers, M.: Van den Berg-Emons, R.: Sluis, T.; Bergen, M.; Stam, H. y Bussmann, H. (2008): "Barriers to and facilitators of everyday physical activity in persons with a spinal cord injury after discharge from the rehabilitation center". Journal of Rehabilitation Medicine, 40, 461-467.

Warner, G.C. (1989): Stress, coping and adjustment to spinal cord injury. Tesis doctoral, Universidad Estatal de Nueva York (Dissertation Abstracts International, vol. 50-07B, pág. 1951).

Wineman, N.M., Duran, E.J. y Steiner, R.P. (1994): "A comparative analysis of coping behaviors in person with multiple sclerosis or a spinal cord injury". Research Nursing and Health, 17 (3), 185194. 